\title{
COEXISTENCE OF LONG-RANGE ORDER FOR TWO OBSERVABLES AT FINITE TEMPERATURES
}

\author{
N. MACRIS AND C.-A. PIGUET \\ Institut de Physique Theorique \\ Ecole Polytechnique Fédérale de Lausanne \\ PHB-Ecublens, CH-1015 Lausanne, Switzerland
}

\begin{abstract}
We give a criterion for the simultaneous existence or non existence of two long-range orders for two observables, at finite temperatures, for quantum lattice many body systems. Our analysis extends previous results of G.-S. Tian limited to the ground state of similar models. The proof involves an inequality of Dyson-Lieb-Simon which connects the Duhamel two-point function to the usual correlation function. An application to the special case of the Holstein model is discussed.

Keywords: long-range order; quantum lattice systems; Holstein model
\end{abstract}




\section{INTRODUCTION}

In a recent paper, G.-S. Tian derived a sufficient condition for the simultaneous existence or non existence of long-range order of two observables in the ground state of quantum lattice models [1]. Here we prove a similar criterion for the case of finite temperatures. As will be seen the theorem is quite general, but for concreteness we limit ourselves to a specific class of fermion Hamiltonians given by (1) below. A concrete example concerning the Holstein model is given at the end and an application to the comparison of critical temperatures for systems with more than one order parameter is briefly discussed.

Let us first recall the result of [酉]. Let $H_{\Lambda}$ be a fermion Hamiltonian of the form

$$
H_{\Lambda}=\sum_{\substack{x, y \in \Lambda \\ \sigma=\uparrow, \downarrow}} t_{x y} c_{x \sigma}^{+} c_{y \sigma}+\sum_{x, y \in \Lambda} V_{x y} n_{x} n_{y}+\sum_{x, y \in \Lambda} J_{x y} \boldsymbol{S}_{x} \cdot \boldsymbol{S}_{y}
$$

where $c_{x \sigma}^{+}, c_{y \sigma}$ are creation and annihiliation operators of spin one-half fermions on a $d$-dimensional lattice $\Lambda \in \mathbb{Z}^{d}, n_{x}$ and $\boldsymbol{S}_{x}$ are the usual fermion density and spin at site $x$. We will always assume that the hopping matrix $t_{x y}$ and the two body interactions $V_{x y}$, $J_{x y}$ are integrable. This implies that $\frac{1}{|\Lambda|} \sum_{x, y \in \Lambda}\left|a_{x y}\right|=O(1)$ with respect to $|\Lambda|$, where $a_{x y}=t_{x y}, V_{x y}, J_{x y}$.

Let $\left|\Psi_{0}^{\Lambda}\right\rangle$ be the ground state of $H_{\Lambda}$. If $O_{x}$ is a local operator, i.e. an operator valued function of $\left\{c_{y \sigma}^{\#}, y \in \mathcal{D}_{x}\right\}$ for $\mathcal{D}_{x}$ a bounded neighborhood of $x$ such that $\left|\mathcal{D}_{x}\right|=O(1)$ with respect to the volume $|\Lambda|$, we define the Fourier transform for each vector $k$ in the reciprocal lattice as

$$
\hat{O}_{k}=\frac{1}{\sqrt{|\Lambda|}} \sum_{x \in \Lambda} \mathrm{e}^{-\mathrm{i} k \cdot x} O_{x}
$$

Suppose there exist three local observables $A_{x}, B_{x}, C_{x}$ such that for each $x \in \Lambda$

$$
\left[H_{\Lambda}, A_{x}\right]=\mu B_{x}+\nu C_{x}
$$


where $\mu$ and $\nu$ are two non-vanishing complex numbers. The result of [1] states that if (5) and some additional assumptions (see later on) are satisfied then

$$
\lim _{\Lambda \rightarrow \mathbb{Z}^{d}} \frac{1}{|\Lambda|}\left\langle\Psi_{0}^{\Lambda}\left|\hat{B}_{k}^{+} \hat{B}_{k}\right| \Psi_{0}^{\Lambda}\right\rangle \neq 0
$$

if and only if

$$
\lim _{\Lambda \rightarrow \mathbb{Z}^{d}} \frac{1}{|\Lambda|}\left\langle\Psi_{0}^{\Lambda}\left|\hat{C}_{k}^{+} \hat{C}_{k}\right| \Psi_{0}^{\Lambda}\right\rangle \neq 0
$$

Notice that if (4) and (5) do not vanish they are strictly positive. In fact (4) and (8) express the presence of long-range order for the observables $B_{x}$ and $C_{x}$. Indeed

$$
\frac{1}{|\Lambda|}\left\langle\Psi_{0}^{\Lambda}\left|\hat{O}_{k}^{+} \hat{O}_{k}\right| \Psi_{0}^{\Lambda}\right\rangle=\frac{1}{|\Lambda|^{2}} \sum_{x, y \in \Lambda} \mathrm{e}^{\mathrm{i} k \cdot(y-x)}\left\langle\Psi_{0}^{\Lambda}\left|O_{y}^{+} O_{x}\right| \Psi_{0}^{\Lambda}\right\rangle \leq \frac{1}{|\Lambda|^{2}} \sum_{x, y \in \Lambda}\left|\left\langle\Psi_{0}^{\Lambda}\left|O_{y}^{+} O_{x}\right| \Psi_{0}^{\Lambda}\right\rangle\right|
$$

Therefore if the left-hand side of (6) is strictly positive one must also have

$$
\lim _{|x-y| \rightarrow \infty} \lim _{\Lambda \rightarrow \mathbb{Z}^{d}}\left|\left\langle\Psi_{0}^{\Lambda}\left|O_{y}^{+} O_{x}\right| \Psi_{0}^{\Lambda}\right\rangle\right| \geq \epsilon>0
$$

for some $\epsilon>0$.

The result of [四] is based on the following inequality which follows from the commutator relation (3). For any $k$

$$
\begin{aligned}
\left(|\mu| \sqrt{\left\langle\Psi_{0}^{\Lambda}\left|\hat{B}_{k}^{+} \hat{B}_{k}\right| \Psi_{0}^{\Lambda}\right\rangle}-|\nu| \sqrt{\left\langle\Psi_{0}^{\Lambda}\left|\hat{C}_{k}^{+} \hat{C}_{k}\right| \Psi_{0}^{\Lambda}\right\rangle}\right)^{2} \leq & \left\langle\Psi_{0}^{\Lambda}\left|\left[\hat{A}_{k}^{+}, \hat{K}_{k}\right]\right| \Psi_{0}^{\Lambda}\right\rangle^{1 / 2} \\
& \cdot\left\langle\Psi_{0}^{\Lambda}\left|\left[\hat{K}_{k}^{+},\left[H, \hat{K}_{k}\right]\right]\right| \Psi_{0}^{\Lambda}\right\rangle^{1 / 2}
\end{aligned}
$$

where $\hat{K}_{k}=\left[H_{\Lambda}, \hat{A}_{k}\right]$. If the right hand side of (\&) is $O(1)$ uniformly in $|\Lambda|$, the two terms in the square are necessarily of the same order. More precisely assuming that the right-hand side is $O(1)$, if one of the correlation functions is $O(|\Lambda|)$, this is also the case for the other. The reader is referred to [2] for several concrete examples. Let us note that if $\nu$ vanishes (resp. $\mu$ vanishes) (8) implies that $\left\langle\Psi_{0}^{\Lambda}\left|\hat{B}_{k}^{+} \hat{B}_{k}\right| \Psi_{0}^{\Lambda}\right\rangle=O(1)$ for all $k$ 
which means there is no long-range order for $B_{x}$ (resp. $C_{x}$ ) [3]. In the next section we prove a similar criterion for the case of finite temperatures.

\section{Finite temperatures}

Let $\left\langle O_{x}\right\rangle_{\Lambda}=\operatorname{Tr}\left(O_{x} \mathrm{e}^{-\beta\left(H_{\Lambda}-\mu N_{\Lambda}\right)}\right) / Z_{\Lambda}$ where the trace is over the usual Fock space, $N_{\Lambda}=\sum_{x \in \Lambda} n_{x}$ and $Z_{\Lambda}=\operatorname{Tr} \mathrm{e}^{-\beta H}$ is the partition function at inverse temperatures $\beta$. The generalisation of (8) to finite temperature involves the Duhamel two-point function whose definition we recall here. The Duhamel two-point function of two operators $F$ and $G$ is defined by

$$
(F, G)_{\Lambda}=\frac{1}{Z_{\Lambda}} \int_{0}^{1} \mathrm{~d} v \operatorname{Tr}\left(\mathrm{e}^{-v \beta\left(H_{\Lambda}-\mu N_{\Lambda}\right)} F \mathrm{e}^{-(1-v) \beta\left(H_{\Lambda}-\mu N_{\Lambda}\right)} G\right)
$$

It is symmetric $(F, G)_{\Lambda}=(G, F)_{\Lambda}$ and satisfies a Cauchy-Schwarz inequality

$$
\left|(F, G)_{\Lambda}\right|^{2} \leq\left(F^{+}, F\right)_{\Lambda}\left(G^{+}, G\right)_{\Lambda}
$$

We also recall the following useful identity

$$
\langle[F, G]\rangle_{\Lambda}=\left(\left[F, \beta H_{\Lambda}\right], G\right)_{\Lambda}
$$

The following lemma generalizes the inequality (8).

\section{Lemma}

Let $A_{x}, B_{x}$ and $C_{x}$ be three local observables satisfying (3) then for all $k$

$$
\left.\left(|\mu| \sqrt{\left(\hat{B}_{k}^{+}, \hat{B}_{k}\right.}\right)_{\Lambda}-|\nu| \sqrt{\left(\hat{C}_{k}^{+}, \hat{C}_{k}\right)_{\Lambda}}\right)^{2} \leq \frac{1}{\beta}\left\langle\left[\hat{A}_{k}^{+}, \hat{K}_{k}\right]\right\rangle_{\Lambda}
$$

\section{Proof:}

Setting $F=\hat{A}_{k}^{+}$and $G=\left[H_{\Lambda}, \hat{A}_{k}\right]=\hat{K}_{k}$ in (11)

$$
\frac{1}{\beta}\left\langle\left[\hat{A}_{k}^{+},\left[H_{\Lambda}, \hat{A}_{k}\right]\right]\right\rangle_{\Lambda}=\left(\left[\hat{A}_{k}^{+}, H_{\Lambda}\right],\left[H_{\Lambda}, \hat{A}_{k}\right]\right)_{\Lambda}=\left(\left[H_{\Lambda}, \hat{A}_{k}\right]^{+},\left[H_{\Lambda}, \hat{A}_{k}\right]\right)_{\Lambda}
$$


Replacing the Fourier transform of (3) in the right-hand side of (13) we obtain

$$
\begin{aligned}
\frac{1}{\beta}\left\langle\left[\hat{A}_{k}^{+}, \hat{K}_{k}\right]\right\rangle_{\Lambda}= & \left(\mu^{*} \hat{B}_{k}^{+}+\nu^{*} \hat{C}_{k}^{+}, \mu \hat{B}_{k}+\nu \hat{C}_{k}\right)_{\Lambda}= \\
& |\mu|^{2}\left(\hat{B}_{k}^{+}, \hat{B}_{k}\right)_{\Lambda}+|\nu|^{2}\left(\hat{C}_{k}^{+}, \hat{C}_{k}\right)_{\Lambda}+\mu^{*} \nu\left(\hat{B}_{k}^{+}, \hat{C}_{k}\right)_{\Lambda}+\nu^{*} \mu\left(\hat{C}_{k}^{+}, \hat{B}_{k}\right)_{\Lambda}
\end{aligned}
$$

We can estimate the last two terms on the right-hand side of (14) using the CauchySchwarz inequality

$$
\begin{array}{r}
\left|\mu^{*} \nu\left(\hat{B}_{k}^{+}, \hat{C}_{k}\right)_{\Lambda}+\nu^{*} \mu\left(\hat{C}_{k}^{+}, \hat{B}_{k}\right)_{\Lambda}\right| \leq|\mu \nu|\left(\left|\left(\hat{B}_{k}^{+}, \hat{C}_{k}\right)_{\Lambda}\right|+\left|\left(\hat{C}_{k}^{+}, \hat{B}_{k}\right)_{\Lambda}\right|\right) \\
\quad \leq 2|\mu \nu| \sqrt{\left(\hat{B}_{k}^{+}, \hat{B}_{k}\right)_{\Lambda}} \sqrt{\left(\hat{C}_{k}^{+}, \hat{C}_{k}\right)_{\Lambda}}
\end{array}
$$

Noticing that the term $\mu^{*} \nu\left(\hat{B}_{k}^{+}, \hat{C}_{k}\right)_{\Lambda}+\nu^{*} \mu\left(\hat{C}_{k}^{+}, \hat{B}_{k}\right)_{\Lambda}$ is in fact real, we have

$$
\mu^{*} \nu\left(\hat{B}_{k}^{+}, \hat{C}_{k}\right)_{\Lambda}+\nu^{*} \mu\left(\hat{C}_{k}^{+}, \hat{B}_{k}\right)_{\Lambda} \geq-2|\mu \nu| \sqrt{\left(\hat{B}_{k}^{+}, \hat{B}_{k}\right)_{\Lambda}} \sqrt{\left(\hat{C}_{k}^{+}, \hat{C}_{k}\right)_{\Lambda}}
$$

From (14) and (16) we get

$$
\frac{1}{\beta}\left\langle\left[\hat{A}_{k}^{+}, \hat{K}_{k}\right]\right\rangle_{\Lambda} \geq\left(|\mu| \sqrt{\left(\hat{B}_{k}^{+}, \hat{B}_{k}\right)_{\Lambda}}-|\nu| \sqrt{\left(\hat{C}_{k}^{+}, \hat{C}_{k}\right)_{\Lambda}}\right)^{2}
$$

which is the desired inequality. To extract information about the correlation functions of $B_{x}$ and $C_{x}$ we have to connect these to the Duhamel two-point function. This will be done through upper and lower bounds on the Duhamel two-point function which involves only the symmetrized correlation function because the Duhamel two-point function is symmetric. The upper bound follows from convexity

$$
\left(F^{+}, F\right)_{\Lambda} \leq \frac{1}{2}\left\langle F^{+} F+F F^{+}\right\rangle_{\Lambda}
$$

The lower bound was found by Dyson, Lieb and Simon [⿴囗十

$$
\left(F^{+}, F\right)_{\Lambda} \geq \frac{1}{2}\left\langle F^{+} F+F F^{+}\right\rangle_{\Lambda} f\left(h_{\Lambda}(F)\right)
$$


where

$$
h_{\Lambda}(F)=\frac{\left\langle\left[F^{+},\left[\beta H_{\Lambda}, F\right]\right]\right\rangle_{\Lambda}}{2\left\langle F^{+} F+F F^{+}\right\rangle_{\Lambda}}=
$$

and the function $f(u)$ is defined implicitely for $u>0$ by

$$
f(u \tanh u)=\frac{1}{u} \tanh u
$$

The function $f(u)$ is continuous convex and strictly decreasing with $\lim _{u \rightarrow 0} f(u)=1$ and $\lim _{u \rightarrow \infty} f(u)=0$.

For any local observable $O_{x}$, we set

$$
O_{\Lambda}(k)=\frac{1}{2}\left\langle\hat{O}_{k}^{+} \hat{O}_{k}+\hat{O}_{k} \hat{O}_{k}^{+}\right\rangle_{\Lambda}
$$

From (12) we get the two inequalities

$$
\begin{aligned}
& |\mu|\left(\hat{B}_{k}^{+}, \hat{B}_{k}\right)_{\Lambda}^{1 / 2} \geq|\nu|\left(\hat{C}_{k}^{+}, \hat{C}_{k}\right)_{\Lambda}^{1 / 2}-\frac{1}{\sqrt{\beta}}\left\langle\left[\hat{A}_{k}^{+}, \hat{K}_{k}\right]\right\rangle_{\Lambda}^{1 / 2} \\
& |\nu|\left(\hat{C}_{k}^{+}, \hat{C}_{k}\right)_{\Lambda}^{1 / 2} \geq|\mu|\left(\hat{B}_{k}^{+}, \hat{B}_{k}\right)_{\Lambda}^{1 / 2}-\frac{1}{\sqrt{\beta}}\left\langle\left[\hat{A}_{k}^{+}, \hat{K}_{k}\right]\right\rangle_{\Lambda}^{1 / 2}
\end{aligned}
$$

Using the upper and lower bounds (18) and (19) we have

$$
\begin{aligned}
& |\mu| B_{\Lambda}(k)^{1 / 2} \geq|\nu| C_{\Lambda}(k)^{1 / 2} f\left(h_{\Lambda}\left(\hat{C}_{k}\right)\right)^{1 / 2}-\frac{1}{\sqrt{\beta}}\left\langle\left[\hat{A}_{k}^{+}, \hat{K}_{k}\right]\right\rangle_{\Lambda}^{1 / 2} \\
& |\nu| C_{\Lambda}(k)^{1 / 2} \geq|\mu| B_{\Lambda}(k)^{1 / 2} f\left(h_{\Lambda}\left(\hat{B}_{k}\right)\right)^{1 / 2}-\frac{1}{\sqrt{\beta}}\left\langle\left[\hat{A}_{k}^{+}, \hat{K}_{k}\right]\right\rangle_{\Lambda}^{1 / 2}
\end{aligned}
$$

From the two last inequalities and the behavior of $f(u)$ for $u \rightarrow 0$, it is easy to deduce the following

\section{Theorem}

Assume there exist three local observables $A_{x}, B_{x}, C_{x}$ satisfying (3). Suppose also that for a given $k$, there exist three positive constants $a_{k}, b_{k}$ and $c_{k}$ independent of $|\Lambda|$ such 
$\operatorname{that}\left\langle\left[\hat{A}_{k}^{+},\left[H_{\Lambda}, \hat{A}_{k}\right]\right]\right\rangle_{\Lambda} \leq a_{k},\left\langle\left[\hat{B}_{k}^{+},\left[H_{\Lambda}, \hat{B}_{k}\right]\right]\right\rangle_{\Lambda} \leq b_{k}$ and $\left\langle\left[\hat{C}_{k}^{+},\left[H_{\Lambda}, \hat{C}_{k}\right]\right]\right\rangle_{\Lambda} \leq c_{k}$. Then for any temperature $\beta^{-1}$

$$
\lim _{\Lambda \rightarrow \mathbb{Z}^{d}} \frac{1}{|\Lambda|} B_{\Lambda}(k) \neq 0
$$

if and only if

$$
\lim _{\Lambda \rightarrow \mathbb{Z}^{d}} \frac{1}{|\Lambda|} C_{\Lambda}(k) \neq 0
$$

In the last theorem, it is important that the expectation values of the double commutators are $O(1)$ with respect to the volume. In the Appendix, we show that this is indeed the case for the Hamiltonian (11) and a large class of observables. It turns out however that this assumption can often be checked explicitely in concrete applications.

\section{Applications}

We wish to present an illustration of the theorem for the dynamic Holstein model. This is a model for molecular crystals which retains the interaction of itinerant electrons with breathing modes of the molecules. These modes are described by Einstein oscillators of frequency $\omega$ attached to each site $x \in \Lambda$. Let $q_{x}, p_{x}$ be the position and momentum of the oscillators (with $\left[q_{x}, p_{y}\right]=\mathrm{i} \delta_{x y}$ ). The Hamiltonian of the model is

$$
H_{\Lambda}=\sum_{x, y \in \Lambda} t_{x y} c_{x}^{+} c_{y}+U \sum_{x \in \Lambda} q_{x}\left(n_{x}-\frac{1}{2}\right)+\frac{1}{2} \sum_{x \in \Lambda}\left(p_{x}^{2}+\omega^{2} q_{x}^{2}\right)
$$

Choosing $A_{x}=p_{x}$ we obtain

$$
\left[H_{\Lambda}, p_{x}\right]=\mathrm{i} U\left(n_{x}-\frac{1}{2}\right)+\mathrm{i} \omega^{2} q_{x}
$$

so that $B_{x}=\left(n_{x}-\frac{1}{2}\right), C_{x}=q_{x}, \mu=\mathrm{i} U$ and $\nu=\mathrm{i} \omega^{2}$. To apply the theorem we have to check that $\left\langle\left[\hat{O}_{k}^{+},\left[H_{\Lambda}, \hat{O}_{k}\right]\right]\right\rangle_{\Lambda}=O(1)$ for $\hat{O}_{k}=\hat{A}_{k}, \hat{B}_{k}$ and $\hat{C}_{k}$. By explicit calculation we 
have

$$
\begin{aligned}
& {\left[\hat{A}_{k}^{+},\left[H_{\Lambda}, \hat{A}_{k}\right]\right]=\omega^{2}} \\
& {\left[\hat{B}_{k}^{+},\left[H_{\Lambda}, \hat{B}_{k}\right]\right]=\frac{1}{|\Lambda|} \sum_{x, y \in \Lambda} 2(\cos (k \cdot(x-y))-1) t_{x y} c_{x}^{+} c_{y}} \\
& {\left[\hat{C}_{k}^{+},\left[H_{\Lambda}, \hat{C}_{k}\right]\right]=1}
\end{aligned}
$$

The double commutators with $\hat{A}_{k}$ and $\hat{C}_{k}$ are constant and thus equal to their expectation values. For $\hat{B}_{k}$ we have

$\left|\left\langle\left[\hat{B}_{k}^{+},\left[H_{\Lambda}, \hat{B}_{k}\right]\right]\right\rangle\right| \leq \frac{1}{|\Lambda|} \sum_{x, y \in \Lambda}|2(\cos (k \cdot(x-y))-1)|\left|t_{x y}\right|\left|\left\langle c_{x}^{+} c_{y}\right\rangle\right|_{\Lambda} \leq \frac{4}{|\Lambda|} \sum_{x, y \in \Lambda}\left|t_{x y}\right|=O(1)$

where we used that for fermionic operators $\left|\left\langle c_{x}^{+} c_{y}\right\rangle\right| \leq 1$. Thus we can conclude that $\lim _{\Lambda \rightarrow \mathbb{Z}^{d}} \frac{1}{|\Lambda|}\left\langle\left(\hat{n}_{-k}-\frac{1}{2}\right)\left(\hat{n}_{k}-\frac{1}{2}\right)\right\rangle_{\Lambda} \neq 0$ if and only if $\lim _{\Lambda \rightarrow \mathbb{Z}^{d}} \frac{1}{|\Lambda|}\left\langle\hat{q}_{-k} \hat{q}_{k}\right\rangle_{\Lambda} \neq 0$.

It follows from this result that if a Peierls instability occurs at a given $k$ (i.e. a distortion of the lattice with long-range order for $q_{x}$ ) there is at the same time longrange order in the electronic density. The occurence of a Peierls instability for $k=\pi$ at low temperature and half-filling has in fact been proven for the static model where the kinetic energy of the phonons in (29) is absent [6]. The present analysis remains unchanged in the static case because even if the term $p_{x}^{2}$ is absent from the Hamiltonian we still have the right to choose $A_{x}=p_{x}$ so that (30) remains unchanged.

In one dimension, for the spinless case the decay of the fermionic correlation functions for the ground state has been analyzed rigorously [0] at weak coupling $U$ by using renormalisation group techniques. The results of [5] imply that these correlations decay algebraically and therefore we can conclude that no long-range order is present at the level of $q_{x}$ as well.

An interesting application of our theorem concerns the comparison of critical temperatures in systems with several types of degrees of freedom and several order parameters. 
An example is the Holstein model itself. Another example are the so-called ferrofluids where one has magnetic (spin) degrees of freedom as well as translational degrees of freedom (see for example [8, 9]). A relevant question is whether there exist two different critical temperatures associated to each order parameters or only one critical temperature where both order parameters simultaneously develop a non zero value. For example in the ferrofluids, it is of interest to decide whether liquid-vapor and ferro-paramagnetic phase transitions occur at the same critical temperature or not. Although our theorem is a statement about existence of long-range order rather than about the order parameter, it suggests that when it is applicable there is only one critical temperature. This fact had been proven earlier for the particular case of the static Holstein model in [6].

A related question that our basic result leaves open is whether the behavior of the two correlation functions at a critical point is governed by two independent length scales. Our discussion above suggests that (when our result is applicable) this is not the case and there is a single diverging length scale involved in both correlations.

\section{Acknowledgements}

We would like to thank C. Gruber, J. Ruiz and V. A. Zagrebnov for discussions. The work of C.-A. Piguet was supported by the Swiss National Science Foundation.

\section{APPENDix}

In order to apply our theorem, it is necessary that all the expectation values of the double commutators are $O(1)$. In this appendix, we check that this is the case for the Hamiltonian (11) and a large class of observables. The double commutators we have to discuss are

$$
\left\langle\left[\hat{O}_{k}^{+},\left[H_{\Lambda}, \hat{O}_{k}\right]\right]\right\rangle_{\Lambda}=\frac{1}{|\Lambda|} \sum_{z, z^{\prime} \in \Lambda} \mathrm{e}^{\mathrm{i} k \cdot\left(z^{\prime}-z\right)}\left\langle\left[O_{z^{\prime}}^{+},\left[H_{\Lambda}, O_{z}\right]\right]\right\rangle_{\Lambda}
$$


where $O_{z}$ is a local observable centered around $z$ of the form

$$
O_{z}=c_{z+\delta_{1}}^{+} \ldots c_{z+\delta_{N}}^{+} c_{z+\epsilon_{1}} \ldots c_{z+\epsilon_{M}}
$$

where $\delta_{i}, i=1, \ldots, N$ and $\epsilon_{j}, j=1, \ldots, M$ are fixed vectors and $N$ and $M$ are fixed numbers. The subsequent discussion provides a proof that (35) is $O(1)$ when $N+M$ is even. If $N+M$ is odd we have only heuristic arguments presented at the end.

We give the detailed calculation for the kinetic term of (11) (for simplicity we omit spin indices). The interaction terms can be treated in a similar way. Let us evaluate the commutator of $O_{z}$ with the kinetic term

$$
\begin{array}{r}
{\left[\sum_{x, y \in \Lambda} t_{x y} c_{x}^{+} c_{y}, O_{z}\right]=\sum_{i=1}^{N} \sum_{x, y \in \Lambda} t_{x y} \delta_{y, z+\delta_{i}} c_{z+\delta_{1}}^{+} \ldots c_{z+\delta_{i-1}}^{+} c_{x}^{+} c_{z+\delta_{i+1}}^{+} \ldots c_{z+\delta_{N}}^{+} c_{z+\epsilon_{1}} \cdots c_{z+\epsilon_{M}}} \\
-\sum_{j=1}^{M} \sum_{x, y \in \Lambda} t_{x y} \delta_{x, z+\epsilon_{j}} c_{z+\delta_{1}}^{+} \ldots c_{z+\delta_{N}}^{+} c_{z+\epsilon_{1}} \ldots c_{z+\epsilon_{j-1}} c_{y} c_{z+\epsilon_{j+1}} \ldots c_{z+\epsilon_{M}}
\end{array}
$$

The commutator of $\mathrm{O}_{z^{\prime}}^{+}$with the first term of the right-hand side of the last equation reads

$$
\begin{aligned}
& \sum_{i=1}^{N} \sum_{x, y \in \Lambda} t_{x y} \delta_{y, z+\delta_{i}}\left(c_{z^{\prime}+\epsilon_{M}}^{+} \ldots c_{z^{\prime}+\epsilon_{1}}^{+} c_{z^{\prime}+\delta_{N}} \ldots c_{z^{\prime}+\delta_{1}} c_{z+\delta_{1}}^{+} \ldots c_{z+\delta_{i-1}}^{+} .\right. \\
& c_{x}^{+} c_{z+\delta_{i+1}}^{+} \ldots c_{z+\delta_{N}}^{+} c_{z+\epsilon_{1}} \ldots c_{z+\epsilon_{M}} \\
& \left.-c_{z+\delta_{1}}^{+} \ldots c_{z+\delta_{i-1}}^{+} c_{x}^{+} c_{z+\delta_{i+1}}^{+} \ldots c_{z+\delta_{N}}^{+} c_{z+\epsilon_{1}} \ldots c_{z+\epsilon_{M}} c_{z^{\prime}+\epsilon_{M}}^{+} \ldots c_{z^{\prime}+\epsilon_{1}}^{+} c_{z^{\prime}+\delta_{N}} \ldots c_{z^{\prime}+\delta_{1}}\right)
\end{aligned}
$$

Using the fermionic commutation relations, we can drive in both terms the creation operators to the left and the annihiliation operators to the right. Doing this we obtain a finite number of terms involving two Kronecker symbols of the type $\delta_{y, z+\delta_{i}} \delta_{z^{\prime}+\mu, z}$ or $\delta_{y, z+\delta_{i}} \delta_{z^{\prime}+\mu, x}$, with $\mu$ a linear combination of $\delta$ 's and $\epsilon$ 's, plus a supplementary term 
containing only one Kronecker symbol. The latter one is

$$
\begin{array}{r}
(-1)^{N^{2}}\left(1-(-1)^{(M+N)^{2}}\right) \sum_{i=1}^{N} \sum_{x, y \in \Lambda} t_{x y} \delta_{y, z+\delta_{i}}\left(c_{z^{\prime}+\epsilon_{M}}^{+} \ldots c_{z^{\prime}+\epsilon_{1}}^{+} c_{z+\delta_{1}}^{+} \ldots c_{z+\delta_{i-1}}^{+} c_{x}^{+} .\right. \\
\left.c_{z+\delta_{i+1}}^{+} \ldots c_{z+\delta_{N}}^{+} c_{z^{\prime}+\delta_{N}} \ldots c_{z^{\prime}+\delta_{1}} c_{z+\epsilon_{1}} \ldots c_{z+\epsilon_{M}}\right)
\end{array}
$$

We can now replace these expressions into (35). Let us first suppose that $M+N$ is even. In this case the term (39) vanishes and only the terms with two Kronecker symbols contribute. It follows from the previous discussion that the contribution of (38) to (35) is bounded by

$$
\frac{1}{|\Lambda|} \sum_{i=1}^{N} \sum_{x, y, z, z^{\prime} \in \Lambda} \Delta_{i}\left(x, y, z, z^{\prime}\right)\left|t_{x y}\right|\left|\left\langle\ldots c^{+} \ldots c \ldots\right\rangle_{\Lambda}\right|
$$

where $\Delta_{i}\left(x, y, z, z^{\prime}\right)$ is one of the following: $\delta_{y, z+\delta_{i}} \delta_{z^{\prime}+\mu, z}$ or $\delta_{y, z+\delta_{i}} \delta_{z^{\prime}+\mu, x}$. The expectation value of the product of $c^{\#}$ can be bounded by 1 since the norm of the fermionic operators is less than 1 . Then using the integrability of $t_{x y}$, the two Kronecker symbols and the factor $1 /|\Lambda|$, we conclude that (40) is bounded by some constant uniformly in the volume.

It is possible to perform a similar discussion for the second term in the right-hand side of (37) to conclude that the double commutators of local observables of the type (36) with $H$ have to be $O(1)$ with respect to the volume in the case $N+M$ even.

In the case where $N+M$ is odd, the discussion for the terms with two Kroneckers symbols is the same as in the case of $N+M$ even. However, there remains the term (39). Replacing it in (35) produces a term bounded by

$$
\begin{array}{r}
\frac{1}{|\Lambda|} \sum_{i=1}^{N} \sum_{x, y, z, z^{\prime} \in \Lambda} \delta_{y, z+\delta_{i}}\left|t_{x y}\right| \mid\left\langle c_{z^{\prime}+\epsilon_{M}}^{+} \ldots c_{z^{\prime}+\epsilon_{1}}^{+} c_{z+\delta_{1}}^{+} \ldots c_{z+\delta_{i-1}}^{+} c_{x}^{+} c_{z+\delta_{i+1}}^{+} \ldots c_{z+\delta_{N}}^{+}\right. \\
\left.c_{z^{\prime}+\delta_{N}} \ldots c_{z^{\prime}+\delta_{1}} c_{z+\epsilon_{1}} \ldots c_{z+\epsilon_{M}}\right\rangle_{\Lambda} \mid
\end{array}
$$

In this last expression, the averages of the product of creation and annihiliation operators are matrix elements of the reduced density matrix $\rho_{M+N}$. In [7] Yang conjectured that 
long range correlations (ODLRO) may appear in such matrix elements only for $N+M$ even. We are not aware of a general proof of this conjecture but it suggests that the matrix element in (41) decays as $\left|x-z^{\prime}\right| \rightarrow \infty$. Assuming the decay is quick enough the sum over $z^{\prime}$ in (41) converges, which then leads to an upper bound

$$
\frac{c}{|\Lambda|} \sum_{i=1}^{N} \sum_{x, y, z \in \Lambda} \delta_{y, z+\delta_{i}}\left|t_{x y}\right|
$$

where $c$ is a constant independent of the volume. Summing over $z$, using the integrability of the hopping amplitudes and the term $1 /|\Lambda|$, we have that (42), and thus (41), are bounded by a constant uniformly in the volume. 


\section{REFERENCES}

[1] Tian G S 1997 J. Phys. A: Math. Gen. 30841

[2] Tian G S 1994 J. Phys. A: Math. Gen. 27 4043; 1994 J. Phys. A: Math. Gen. 27 6677; 1997 Phys. Rev. B 565355

[3] Tian G S 1993 J. Phys. A: Math. Gen. 26 2325; 1994 Solid State Commun. 90837

[4] Dyson F J, Lieb E H and Simon B 1978 J. Stat. Phys. 18335

[5] Benfatto G, Gallavotti G and Lebowitz J L 1995 Helv. Phys. Acta 68312

[6] Lebowitz J L and Macris N 1994 J. Stat. Phys. 7691

[7] Yang C N 1962 Rev. Mod. Phys. 34694

[8] Gruber C and Griffiths B 1986 Physica A 138220

[9] Romano S and Zagrebnov V A 1998 Physica A 253483 